\title{
A CONTRIBUTION TO THE PSYCHOPATHOLOGY OF RESIDUAL ENCEPHALITIS LETHARGICA.
}

\author{
By C. WORSTER-DROUGHT AND D. N. HARDCASTLE, LoNdoN.
}

RECEnT literature dealing with the residua of encephalitis lethargica tends rather to emphasize cortical cell damage as responsible for the psychological disturbances that may result from the disorder. In dealing with many cases of the so-called sequelæ of encephalitis lethargica (more properly the chronic stage), it appeared to us that the patients showed little or no impairment of intellect, and that the apathy -which is often so predominant a feature-was largely a volitional disorder associated with considerable retardation along the efferent paths (e.g., of speech, expression, etc.) below the cortical level.

With the object of testing the above hypothesis, we subjected a series of nineteen cases, together with normal controls, to two sets of investigations. In each set the patient's time-reaction was ascertained by a visual stimulus consisting in the lighting of a small electric lamp. With stimuli of the same nature, a further time-reaction that involved a selection was also determined. We thus obtained :--

1. The psychomotor reaction-time;

2. The cerebration time for selection-by subtracting the psychomotor reaction-time from the figure obtained for the total period occupied in the reception of the stimulus, the selection, and the indication of the choice.

Of the nineteen cases investigated, seventeen patients exhibited some form of 'Parkinsonian syndrome,' the degree of which varied in different cases from relative facial immobility to complete symptomatic paralysis agitans. The remaining two suffered from residual cranial nerve palsies, and one, in addition, from spastic paraplegia. The condition had originated during 1918 in four cases, during 1919 in five cases, during 1920 in five cases, during 1921 in four cases, and during 1922 in one case. At least nine of the cases exhibited a progressive type of the disease.

For the purpose of the investigation we used a specially designed apparatus (devised by D. N. H.) comprising four press-buttons connected to two electrically controlled switches, two small electric light bulbs, and a stop-watch inserted into the light circuit. In front of the operator were placed the stop-watch, the switches, and the two press-buttons 
which lighted the right and left lamps respectively and also started the stop-watch. The patient was seated in front of the two lamps, and in each hand held a press-button (the second pair) which, on being pressed, turned out the right and left lamp respectively, and also stopped the stop-watch. In the patient's press-button circuit two small 'tell-tale' lamps were fixed on the switch-box, in order to indicate to the operator which button the patient had pressed (see diagram).

\section{Psychomotor Reaction-time.}

Simple time-reactions were first determined with the patient's right hand and then with the left; these were repeated, and a mean for each hand was taken. Thus the psychomotor reaction-time was obtained for a single visual stimulus for each hand, every care being taken to avoid rhythm.

\section{Cerebration Time for Selection.}

(a) The patient was told that either the right or left lamp would be lighted, and that he had to respond by pressing the appropriate button controlling the lighted lamp (and thus extinguishing it). It was, therefore, necessary for him to make a choice of the press-buttons. Again the reactions were repeated and a mean obtained. Deceit or errors on the part of the patient were revealed by the 'tell-tale' lamps described above.

(b) The two lamps used for the visual stimulus were then removed, and a box, containing an electric light placed behind a slide-holder (slide box), was substituted in their place. Into the slide-holder could be dropped translucent slides carrying figures or letters which were at once illuminated by switching on the light. Two sets of slides-each comprising twelve slides-were employed, and were presented to the patient in a definitely irregular order.

Set 1.-The slides showed simple addition sums, six of which were correctly totalled and six incorrectly.

Set 2.-The slides showed a number of capital E's irregularly scattered in a line of other capitals; on each slide was also a figure showing in six of the slides the correct number of capital E's and in six an incorrect number.

The patient, still holding with respective hands the right and left press-buttons (which now controlled the single light behind the translucent slide), was instructed to work out the solution presented, and, if correctly stated on the slide, to press the right button, or, if incorrectly stated, the left button. The lamp illuminating the slide was turned on by the operator, the same switch starting the stop-watch; this latter was stopped only when the patient pressed the button indicating a 
correct answer. The patient's time for each of the twelve slides was taken.

The results of these investigations were as follows :-

1. Psychomotor Reaction-time.-The reaction-time for the single visual stimulus, as compared with a number of normal controls, was considerably lengthened in the cases of residual encephalitis. In the normal subjects - with the same apparatus - the average reaction-time was 0.24 seconds, while in the nineteen patients the average time was 0.36 seconds. Only three of the patients reacted within normal limits.

2. The Cerebration Time.-(a) In the ' choice of light' the cerebration time of only three patients exceeded the figures obtained in normal subjects, while the average time in the nineteen patients was actually slightly less than the average in the normal controls. In the latter the average cerebration time was $0 \cdot 18$ seconds, while that for the nineteen encephalitic cases was $0 \cdot 14$ seconds.

(b) In the 'slide' investigations the result was as follows :-

Set 1 (addition sums).- The average cerebration time for the nineteen patients was slightly shorter $(2 \cdot 6$ seconds $)$ than in the normal subjects $(2 \cdot 9$ seconds), but the former were less accurate, 60 per cent. of patients showing 8.5 per cent. errors as compared with 25 per cent. of normal subjects showing 6 per cent. errors.

Set 2 (capital letters).-The average cerebration time in the nineteen patients was only slightly higher $(4 \cdot 0$ seconds $)$ than in normal subjects (3.7 seconds), but again there was a higher percentage of errors- $\mathbf{7 5}$ per cent. of patients showing 12 per cent. errors compared with 25 per cent. normal controls showing 2 per cent. errors. The mistakes in some of the cases may have been due to organic difficulties in reading the lettersfaulty accommodation or diplopia ; four patients were unable to complete the test owing to such disabilities.

3. General Intelligence Tests.-The next group of tests applied were of the general intelligence order, some of which were taken frcmTerman's Measurement of Intelligence. The nineteen patients were all investigated and compared with normal subjects.

Only three patients fell below the standard adopted from our investigation of normal controls, the deficiency being chiefly under the headings of ' reason' and 'memory.' Two of these patients, however, were aged twelve and thirteen years respectively at the onset of the disease, and had been away from school since that time; the third case was that of an ex-soldier with a profound psychoneurosis in addition to the residual encephalitis. The reaction-times in this series of tests again showed a definite lengthening, averaging two minutes forty seconds for the patients, as compared with an average of one minute forty seconds for normal subjects-viz., reaction-time increased by just over 50 per 
cent., which is the same figure obtained in the simple psychomotor response.

From our observations it would also appear that the sufferer from

Diagram of Apparatus Used.

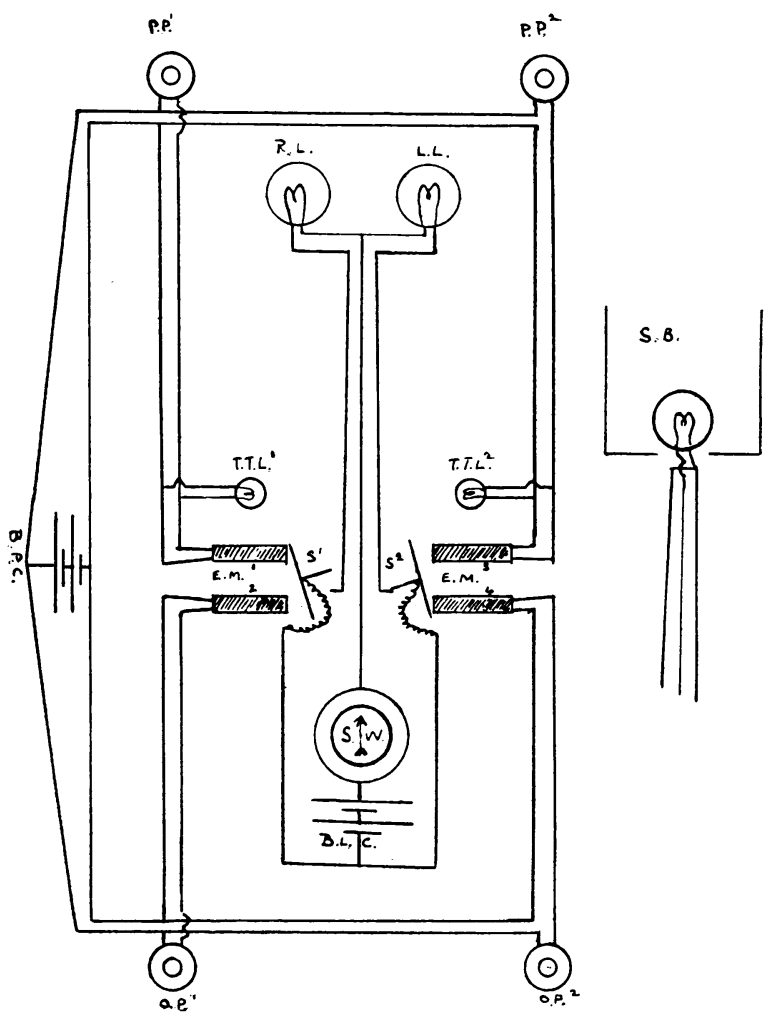

$\mathrm{PP}^{1}$ and $\mathrm{PP}^{2}=\mathrm{R}$ and $\mathrm{L}$ hand patient's press-buttons respectively : $\mathrm{RL}$ and $\mathrm{LL}=\mathrm{R}$ and $\mathrm{L}$ hand patient's stimulus lights respectively; $\mathrm{TTL}^{1}$ and $\mathrm{TTL}^{2}=$ 'Tell-tale' lights in $\mathrm{PP}$ circuits ; $\mathrm{EM}=$ Electromagnets excited by circuits $\mathrm{PP}$ and $\mathrm{OP}$ and operating $S^{1}$ and $S^{2}=R$ and $L$ hand switches. $\left(S^{1}=\right.$ switch in 'off' position ; $\mathrm{S}^{2}=$ switch in 'on' position.) $\mathrm{SW}=$ stop-watch ; $\mathrm{OP}^{1}=$ operator's pressbutton controlling $\mathrm{RL}, \mathrm{OP}^{2}$ controlling $\mathrm{LL} ; \mathrm{BLC}=$ battery light circuit ; $\mathrm{BPC}=$ battery press-button circuit; $\mathrm{SB}=$ slide box. (The two lights RL and LL are removed and the slide box substituted for the second part of the reaction-time experiment.)

Method of Operation.- $\mathrm{OP}^{2}$ on being pressed completes the circuit $\mathrm{EM}^{4}$ and brings $S^{2}$ into 'on' position, making circuit through LL and starting SW. The patient reacting presses $\mathrm{PP}^{2}$, which excites $\mathrm{EM}^{2}$ and at the same time lights the small lamp TTL ${ }^{2}$; $\mathrm{EM}^{3}$ then pulls $\mathrm{S}^{2}$ into 'off' position as shown in $\mathrm{S}^{1}$, extinguishes LL and stops SW.

residual encephalitis lethargica is continually attempting to compensate for his psychomotor retardation, and is thus living in a perpetual state of what may be termed 'increased psychic tension'; this, in turn, affects the patient's relation to his environment (as compared with his 
condition prior to the illness), and tends to bring about manifestations of an ' anxiety' state. In this matter we entirely agree with the findings of Jones and Raphael that "a latent psychoneurotic tendency before the illness may afterwards become apparent." Twelve of our patients suffered from definite psychoneurotic symptoms-apprehension, severe depression, undue self-consciousness, asociability, terror-dreams, etc. Two patients each gave a history of some moral deterioration following closely upon the initial attack, but according to their relatives they have since returned to normal behaviour.

Our conclusions may be summarized as follows: In residual encephalitis lethargica-

1. The psychomotor reaction-time is definitely lengthened-usually by about 50 per cent.

2. The cerebration time is not appreciably affected.

3. Psychoneurotic manifestations are frequent, and are often the result of a latent psychoneurotic tendency rendered manifest by the disease. 